\title{
An extreme weight loss reflective, advice on a healthy life change
}

\section{Perspective}

"Wow, you look so great since I've seen you last! What's your big weight-loss secret?" I've been approached with this question countless times over the past several years, and I always seem to receive the generally disinterested retort of "...oh" when I reveal what my great "cure-all" is. It's hard work and determination. This is not a "magic pill" article, but this diet plan works 100 percent of the time.

My weight-loss journey was not an excessively difficult one, nor was it painful, but it did take me a fair amount of time to get down to a weight at which I was comfortable living. It took me about four years to reach an optimal weight for my 5'9" frame and large body-type, but I lost 170pounds during this process.

This reflection is written in mind of not only those who intend to lose a large sum of weight, but for those who wish to make just a few lifestyle changes as well. Contrary to unproven supplement companies and hokey weight-loss regimens, THERE IS NO MAGIC CURE. There isn't. This will not happen overnight, and it's not something that one can complete and then follow-up by going off to eat whatever they want and remaining skinny. However, this method works every time, and each-and-every person can customize this plan to their exact individual needs.

\section{Step I: shrinking your stomach}

The first (and probably most challenging) step is shrinking the stomach muscle. The intestinal musculature of the stomach is so pliable and strong that it can continuously expand to accommodate the amount of food a person is intaking. For me at my largest (340pounds), this was somewhere in the range of about 3,600calories a day (double what is needed for my body type). Luckily for our firstworld mammalian creatures, the stomach muscle is also able to be manipulated into a much smaller size. (This does not require surgery, although some obesity patients do choose this route.)

The way I accomplished the shrinking of my stomach musculature was through a strict 1,200 calorie per-day diet for the first entire year of my weight loss. I'm sure this doesn't sound like a cake-walk (and no, there wasn't much cake involved), but the only time it was actually very difficult was during the first two months. To satiate my hunger cravings, I drank around 10 8-oz glasses of water per day. I kept lowcalorie snacks around me at all times, such as cucumber, pickles, dry popcorn, low-sodium chicken broth, and baby carrots. I wasn't ever excessively hungry, but I did get frustrated now and again. After all, none of these snacks constitutes pizza. It wasn't all water and celery, however if I really did want pizza, or a piece of cake, I ate it! I kept myself accountable by either subtracting the calories from my daily allowance, or divvying them up via my weekly calorie allowance. If I wanted to subtract pizza calories from just one day (for dinner, for example), that meant canned tuna and raw spinach for breakfast and lunch that day. This is not a diet to keep for many years, as extended use of such a diet will slow the body's metabolism, making it even more difficult to lose future weight. However, this 1,200-calorie
Volume 7 Issue 4 - 2017

\author{
Devin Ramnarain \\ Subclinical Nutritionist, USA
}

Correspondence: Devin Ramnarain, Subclinical Nutritionist, 8I0I SW. 24th Ct, \#305, USA, Tel 4I7489|847,

Email D Ferrell@outlook.com

Received: October II, 2017 | Published: October 23, 2017

regimen granted me my first 100-pounds of weight-loss, in just one year.

\section{Step 2: exercise regimen}

During the first year of my weight-loss, exercise was moderately painful for me to accomplish. Being 340 pounds put a lot of pressure on my joints, heart, and back. I would walk one mile around my neighborhood, twice a week. After my 100-pound loss (now 2401b), cardio-inclusive exercise was more readily possible. I attained a gym membership, and would run on an elliptical for about 15 minutes at a time, three times a week. I paired this with one 1-mile walk per week, and aerobic dancing at home (very good exercise!) I would conduct mild weight training at the gym when I felt ready to (approximately once weekly). Over the next three years, I would continue to gradually increase this regimen as I was ready, moving to 20 minutes and then 30 on the elliptical. At my current level, I am able to comfortably run for an hour on an elliptical four times a week, paired with moderate weight training every time. I frequently include about 8hours of cardio/weight-training into my week, because I allowed myself to gradually get stronger. Gym time is by no means the only method to good exercise. Anything that gets the heart beating can be entered into this sect of my regimen. Some other methods I personally use are lapswimming, trampoline, hiking, and yoga.

\section{Step 3: plan a long-term lifestyle change}

As aforementioned, the 1,200-kilocalorie-per-day diet isn't a lifelong fix. One must realize that in order for the results attained from this jumpstart to be lifelong, a new plan must be put into effect. After year-1, I graduated my caloric allowance to 1,500 for about three months, and then to 1,700 for the following six months. I focused strongly on the dietetic guidelines of My Plate, ensuring that I consumed three servings of each proteins, vegetables, fruits, and dairy per day. My carbohydrate intake was allowed at around 3-6 standard servings. Over the three-years subsequent, I lost another 70 pounds, for a grand-total of 170pounds lost, about half my entire body weight. Nowadays, I eat approximately 2,300calories daily, but I no longer count calories. I eat pizza and donuts, but only when I feel like consuming them (I treat myself about once a week with a muffin or cheeseburger). My body is satisfied with the postulation of vitamins and minerals that I provide it now, that I no longer have a desperate need for excessive sugars, fats, and salts. Eating right is so simple for me now that I seldom even think about it. I eat all the time, huge 
meals, but the food that these meals consist of is all healthy. Staying on track is second-nature to me because I changed my diet over time and psychologically trained myself to be ready to see myself at new physical sizes, and to crave the right foods for my body.

\section{Conclusion}

Take weight-loss slowly, one day at a time. Especially if one is losing a considerable amount of weight, the change should NOT happen overnight. For lasting changes, one must be prepared to MAKE lasting changes in their lifestyle. For example, I went from a 340-pound teenager with pre-diabetes, prehypertension, and a pinched nerve near my lower spine from obesity to a 170-pound young adult pursuing nutrition as my life's work. I have spoken at colleges and started a site to help others gain the confidence to make this life change. There is no magical cure for weight loss. Even with surgery - if one makes poor choices subsequent to the operation, they will be in the same (or an even worse) situation. It's just like reversing bad credit: It takes patience, determination, and sometimes doing things that aren't the easiest or most fun choices at the time. If one stays strong, keeps focused on the end result, and doesn't throw up their hands when they have an accidental cheat-day, any weight goal is achievable.

\section{Acknowledgements}

None.

\section{Conflict of interest}

The author declares no conflict of interest. 